\title{
Metastasis of Pulmonary Adenocarcinoma presenting as Multiple Cystic Lesions of the Brain: Importance of Differential Diagnosis
}

\author{
Dragan Janković ${ }^{1}$, Harald Krenzlin ${ }^{1}$, Naureen Keric ${ }^{1}$, and Florian Ringel ${ }^{1}$ \\ ${ }^{1}$ University Medical Centre of the Johannes Gutenberg University Mainz
}

March 27, 2021

\begin{abstract}
A 62-year-old otherwise healthy patient was admitted with severe headache for several weeks. Initial imaging studies revealed multiple cystic lesions in both hemispheres. Neuroparasitic infections were considered as primary differential diagnosis. Open biopsy of a right frontal cortical lesion was performed. Pathohistological revealed the diagnosis of cerebral metastasis of adenokarcinoma.
\end{abstract}

Metastasis of Pulmonary Adenocarcinoma presenting as Multiple Cystic Lesions of the Brain: Importance of Differential Diagnosis

Dragan Jankovic ${ }^{1}$, Harald Krenzlin ${ }^{1}$, Naureen Keric ${ }^{1}$, Florian Ringel ${ }^{1}$

${ }^{1}$ Department of Neurosurgery, University Medical Centre of the Johannes Gutenberg University of Mainz, Mainz, Germany

Corresponding author:

Dragan Jankovic, MD

Department of Neurosurgery

University Medical Center Mainz

Johannes Gutenberg University of Mainz

Langenbeckstr. 1

55131 Mainz, Germany

Phone: +49-6131-177331

Fax: +49-6131-172274

Email: dragan.jankovic@unimedizin-mainz.de

Abstract A 62-year-old otherwise healthy patient was admitted with severe headache for several weeks. Initial imaging studies revealed multiple cystic lesions in both hemispheres. Neuroparasitic infections were considered as primary differential diagnosis. Open biopsy of a right frontal cortical lesion was performed. Pathohistological revealed the diagnosis of cerebral metastasis of adenocarcinoma.

\section{Accompanying text}

Cystic lesion of brain can present in several diseases, such as inflammatory and demyelinating disorders, bacterial and fungal infections, parasitic disease, but also as metastases and primary brain tumor. ${ }^{1}$ 
We present a case of a 62-year-old otherwise healthy male presented with severe headache for a several weeks. Neurologic examination revealed a mild dysdiadochokinesia. Magnetic resonance imaging (MRI) demonstrated supra- and infratentorial disseminated cystic lesions, which lead to generalized cerebral edema. Radiographic appearance was suggestive of neuroparasitic disease, such as Toxoplasmosa, Neurocysticercosis, Echinococcosis.

Routine blood test showed elevated white blood cell counts and normal C-reactive Protein (CRP) values. Blood cultures and screening tests for Toxoplasmosis, Neurocysticercosis, Echinococcosis and Toxocara returned negative. Immunserological findings revealed a relevant increased ANA titer.

As a definitive diagnosis remained elusive, open neuronavigated brain biopsy of right frontal lesion was performed. Histopathological analysis revealed a brain metastasis of an adenocarcinoma. Chest CT revealed a $2,7 \times 1,9 \times 2,3 \mathrm{~cm}$ large tumor in the right lower lobe bronchus.

After the diagnosis was confirmed, the patient underwent whole brain radiotherapy (WBRT), followed by chemotherapy. A follow-up MRI of brain showed clearly decreasing Metastases supra- and infratentorial with decreasing perifocal edema. The patient has no new neurological deficits.

\section{CONFLICT OF INTEREST}

Authors have no conflict of interest to disclose.

Conflict of interest: Authors have no conflict of interest do disclose.

Author contributions: DJ: wrote the manuscript. HK and NK: editing and proofread the manuscript. FR: supervision.

\section{References}

1. Marta GN, da Cunha Colombo Bonadio RR, Martins RE, Zuppani HB, de Castro G Junior. Cystic brain metastases in ALK-rearranged non-small cell lung cancer. Ecancermedicalscience. 2018;12:818.

\section{Figure legends}

Figure 1. T1 magnetic resonance imaging sequences showing a supratentorial and infratentorial multiple lesions with perifocal edema; (a) axial, (b) axial, (c) coronal, (d) sagittal
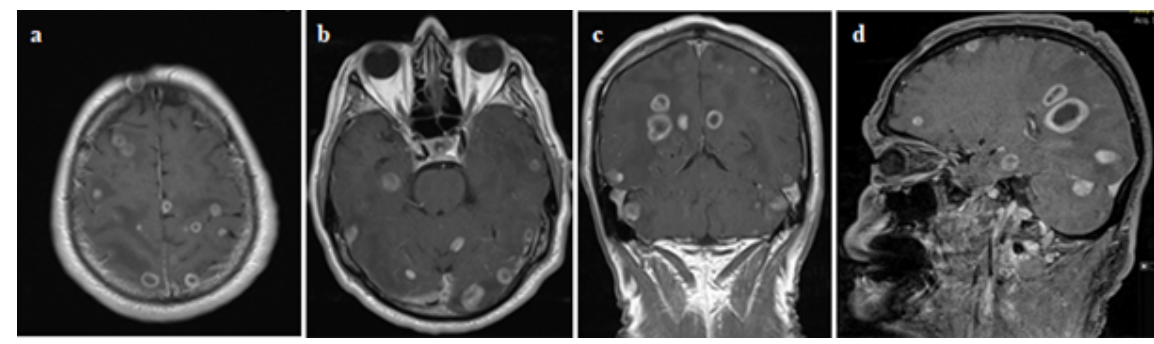